\title{
Is Background Parenchymal Enhancement on Magnetic Resonance Imaging Helpful in Risk Assessment of Breast Cancer?
}

\author{
Maryam Farghadani, ${ }^{1, *}$ Ali Hekmatnia, ${ }^{2}$ Hoda Sharif, ${ }^{2}$ Mehdi Baradaran, ${ }^{2}$ and Maryam Riahinezhad ${ }^{3}$ \\ ${ }^{1}$ Assistant Professor of MUI \\ ${ }^{2}$ Isfahan Medical University \\ ${ }^{3}$ Isfahan University of Medical Sciences \\ "Corresponding author: Maryam Farghadani. E-mail: dr_mar_far@yahoo.co.uk
}

Received 2016 December 21; Accepted 2017 February 08.

\begin{abstract}
Objectives: Background parenchymal enhancement (BPE) on contrast-enhanced magnetic resonance imaging (CE-MRI) corresponds to hormonally active breast fibroglandular tissue. Several studi.es have reported increased odds of breast cancer in patients with marked BPE. The purpose of the present study was to evaluate BPE in contralateral breast of biopsy proven breast cancer patients and comparison of the results with control group.

Methods: In a case-control study, CE-MRI of the contralateral breasts of 40 biopsy-proven breast cancer patients and 40 patients with biopsy- proven benign breast lesions, were retrospectively reviewed by an experienced radiologist and degree of BPE, and pattern of enhancement were determined, finally, BPE was compared between case and control groups using chi square test. Pearson correlation test was used to determine association between FGT amount and BPE. P value $<0.05$ was considered significant.

Results: In the case group, 4, 17,14, and 5 patients had minimal, mild, moderate, and marked BPE, respectively. In the control group, 12, 24, and 4 participants had minimal, mild, and moderate BPE, respectively. BPE was significantly higher in the case group in comparison with the control group (P value $<0.05$ ). Patients with invasive carcinoma had significantly higher BPE in comparison with the patients with carcinoma in situ. (P value $<0.05)$ There was a moderate correlation $(r=0.4$, P value $<0.05)$ between FGT amount and degree of BPE. In the case group, 3, 26, and 11 patients had mass-non mass, mass, and non mass pattern of enhancement, respectively. In the control group, 25, antl15 participants had mass, and non mass pattern of enhancement, respectively. Pattern of enhancement was not significantly different between the two groups.

Conclusions: Our study revealed that patients with the diagnosis of breast cancer have greater BPE in comparison with control group. The results may be indicative for higher probability of developing breast cancer in patients with higher BPE. Therefore, CEMRI could be a useful tool for breast cancer risk assessment.
\end{abstract}

Keywords: Background Enhancement, MRI, High Risk Breast Cancer

This is an abstract presented in the 33rd Iranian congress of radiology (ICR) and the 15th congress of Iranian radiographic science association (IRSA). 